\title{
Potential anti-tumor effect of a nanoliposomal antiPCSK9 vaccine in mice bearing colorectal cancer
}

Amir Abbas Momtazi-Borojeni ${ }^{1,2}$, Maryam Ebrahimi Nik³ ${ }^{3}$ Mahmoud Reza Jaafari ${ }^{4,5}$, Maciej Banach ${ }^{6,7}$, Amirhossein Sahebkar ${ }^{5,8,9}$

\author{
${ }^{1}$ Nanotechnology Research Center, Bu-Ali Research Institute, Mashhad University \\ of Medical Sciences, Mashhad, Iran \\ ${ }^{2}$ Department of Medical Biotechnology, Student Research Committee, \\ Faculty of Medicine, Mashhad University of Medical Sciences, Mashhad, Iran \\ ${ }^{3}$ Nanotechnology Research Center, Student Research Committee, Pharmaceutical \\ Technology Institute, Mashhad University of Medical Sciences, Mashhad, Iran \\ ${ }^{4}$ Nanotechnology Research Center, Pharmaceutical Technology Institute, Mashhad \\ University of Medical Sciences, Mashhad, Iran \\ ${ }^{5}$ Biotechnology Research Center, Pharmaceutical Technology Institute, Mashhad \\ University of Medical Sciences, Mashhad, Iran \\ ${ }^{6}$ Department of Hypertension, WAM University Hospital, Medical University of Lodz, \\ Lodz, Poland \\ 7Polish Mother's Memorial Hospital Research Institute (PMMHRI), Lodz, Poland \\ ${ }^{8}$ Neurogenic Inflammation Research Center, Mashhad University of Medical Sciences, \\ Mashhad, Iran \\ ${ }^{9}$ School of Pharmacy, Mashhad University of Medical Sciences, Mashhad, Iran
}

Arch Med Sci 2019; 15 (3): 559-569

DOI: https://doi.org/10.5114/aoms.2019.84732

Copyright $\odot 2019$ Termedia \& Banach

Submitted: 1 March 2019

Accepted: 5 April 2019

\begin{abstract}
Introduction: Inhibition of proprotein convertase subtilisin/kexin 9 (PCSK9) is an effective therapeutic tool for lowering low-density lipoprotein cholesterol (LDL-C). There is no available evidence on the efficacy and safety of PCSK9 inhibitors in non-cardiovascular diseases, particularly cancer. The present study aimed to evaluate the effect of PCSK9 inhibition on cancer endpoints in mice bearing colon carcinoma, using a nanoliposomal antiPCSK9 vaccine. Material and methods: The prepared nanoliposomal antiPCSK9 vaccine was subcutaneously inoculated in BALB/c mice four times with a biweekly interval. Two weeks after the last booster, the vaccinated and unvaccinated mice were subcutaneously inoculated with CT26 colon cancer cells into the right flank. After the tumor mass became palpable, the mice were randomly divided into three groups: (1) PBS (untreated control), (2) vaccine group, and (3) pegylated liposomal doxorubicin (PLD; positive control) group. Body weight, tumor size and survival of mice were monitored for 50 days.

Results: The nanoliposomal antiPCSK9 vaccine could efficiently provoke specific antibodies against PCSK9 in BALB/C mice and thereby reduced the plasma level and function of PCSK9. Tumor volume was $77 \%$ and $87.7 \%$ lower $(p<0.0001)$ in the vaccinated mice when compared with Doxil (liposomal doxorubicin) and control mice, respectively. Tumor size analysis showed that time to reach the endpoint of the vaccine group ( $47 \pm 11$ days) was slightly but not significantly higher than PLD ( $46 \pm 2.6$ days) and the control $(43 \pm 12$ days) groups. The tumor growth rates in the vaccine and PLD groups were reduced by $9.3 \%$ and 7.3 , respectively, when compared with the control group. The vaccinated mice survived slightly but not significantly longer than PLD and the control mice. The median survival of the vaccine, PLD and control groups were 51, 45, and 41 days, respectively. The vaccinated mice's life was prolonged by $24.4 \%$ as compared with the control mice, while it was increased by $9.8 \%$ in the PLD group.
\end{abstract}

\author{
Corresponding author: \\ Dr. Amirhossein Sahebkar \\ Biotechnology \\ Research Center \\ Pharmaceutical \\ Technology Institute \\ Mashhad University \\ of Medical Sciences \\ 9177948564 Mashhad \\ Iran \\ E-mail: sahebkara@mums. \\ ac.ir; \\ amir_saheb2000@yahoo.com
}


Conclusions: Our results revealed that PCSK9 inhibition not only exerted no harmful effects but also could moderately inhibit tumor growth, and improve lifespan and survival in mice bearing colon cancer.

Key words: colon carcinoma, immunization, liposome, nanoparticle, PCSK9, vaccine.

\section{Introduction}

Abnormal levels of plasma lipids have been shown to be significantly associated with colon carcinoma risk [1-8]. Higher levels of low-density lipoprotein (LDL) cholesterol (LDL-C) have been reported to correlate with higher prevalence $[9,10]$ and risk $[11,12]$ of colon cancer. LDL-C is mainly cleared from the bloodstream by the liver's LDL receptors (LDLRs). Regulation of LDLR expression has an important role in LDL-C hemostasis. Proprotein convertase subtili$\sin /$ kexin 9 (PCSK9) is a liver secretory protein that acts as a key regulator of protein levels of LDLRs, by which plasma levels of LDL-C in the body can be controlled. The secreted PCSK9 circulates in the bloodstream and binds to the cell surface LDLR. PCSK9 binding leads to internalization and degradation of LDLR in the lysosomal compartment and thereby reduces recycling of LDLR to the cell surface $[13,14]$. Consequently, there are not enough LDLRs to remove plasma LDL-C when the circulating level of PCSK9 is increased as a result of gain-of-function mutations [15], whereas low levels of plasma PCSK9, due to loss-of function mutations, will lead to more intact LDLRs which in turn take up more plasma LDL-C [15]. Therefore, PCSK9 inhibition has emerged as a therapeutic tool and is currently used as an effective LDL-lowering approach in hypercholesterolemic patients [16-23]. Although the safety of PCSK9 inhibitors in hypercholesterolemic conditions has been approved, there is scant information on the safety of PCSK9 inhibition in other conditions such as cancer. To date, several human studies have been conducted to evaluate a possible link between PCSK9 gene variants and cancer risk, but the results have been inconsistent. One of the studies showed that there is a significant association between LDL-increasing PCSK9 mutations and a higher risk of cancer [24], while another human study could not show any association between PCSK9 loss-of-function mutations and incidence of cancer [25]. Conversely, a recent Mendelian randomization study showed that PCSK 9 with LDL-increasing mutations is strongly correlated with higher cancer risk, whereas LDL-lowering variants mimicking PCSK9 inhibitors were found to be significantly associated with a lower risk of cancer occurrence [26]. These contradictory reports call for further investigations to assess the safety and efficacy of PCSK9 inhibitors in cancer. Anti-PCSK9 vaccines are a new generation of PCSK9 inhibitors, whose LDL-lowering effect is frequently verified in preclinical studies [27-30]. In a previous study, we reported a nanoliposomal anti-PCSK9 vaccine with long-lasting, specific and safe inhibitory effects on plasma PCSK9 in BALB/c mice [31].

In the current study, we aimed to investigate the effects of PCSK9 inhibition using the a nanoliposomal anti-PCSK9 vaccine in BALB/C mice bearing CT26 colon carcinoma.

\section{Material and methods}

\section{Vaccine preparation and characterization}

\section{Preparation and characterization \\ of the liposome nanoparticles}

The thin film-lipid hydration method was used to synthesize a nanoliposome formulation containing 1,2-dimyristoyl-sn-glycero-3-phosphorylcholine (DMPC), 1,2-dimyristoyl-sn-glycero-3-phosphorylglycerol (DMPG), and cholesterol (Chol) (Avanti Polar Lipid; Alabaster, USA) at a final concentration of $40 \mathrm{mM}$ (total phospholipids and Chol). In brief, DMPC, DMPG, and Chol were mixed in chloroform at the molar ratios of $75: 10: 15$, respectively. Lipid solution was dried to a thin lipid film under reduced pressure using rotary evaporation (Heidolph, Germany), and then the organic solvent was completely eliminated using over-night freeze drying (VD-800F, Taitech, Japan). Afterward, the dried lipids were dispersed using hydration with $10 \mathrm{mM}$ HEPES buffer ( $\mathrm{pH} 7.2$ ) containing 5\% dextrose, followed by vortexing and bath-sonicating to be completely homogenized. The obtained multilamellar vesicles (MLVs) were serially extruded using a mini extruder (Avestin, Canada) with polycarbonate membranes of $600,400,200$, and $100 \mathrm{~nm}$ pore size, respectively, to prepare small unilamellar vesicles (SUVs) with a uniform size of $100 \mathrm{~nm}$. Particle size (diameter, $\mathrm{nm}$ ), zeta potential (surface charge, $\mathrm{mV}$ ) and polydispersity index (PDI) of the prepared nanoliposomal formulation were evaluated using dynamic light scattering (DLS) technique on a Zetasizer (Nano-ZS, Malvern, UK) at room temperature (RT). The prepared liposome nanoparticles were stored at $4^{\circ} \mathrm{C}$ under argon.

\section{Preparation of immunogenic peptide}

The Immunogenic Fused PCSK9-Tetanus (IFPT) peptide with a purity grade of $>95 \%$ was synthe- 
Table I. Sequence of immunogenic peptides used in the present study

\begin{tabular}{|lcl|}
\hline Peptide name & Sequence & Immunogenicity \\
\hline PCSK9 & S-I-P-W-N-L-E-R-I-T-P-V-R & B cell epitope \\
\hline Tetanus & A-Q-Y-I-K-A-N-S-K-F-I-G-I-T-E-L & T cell epitope \\
\hline IFPT & ${ }^{*}$ CGGGSIPWNLERITPVRKKAQYIKANSKFIGITEL & \\
\hline
\end{tabular}

*The bold amino acid codes are a linker sequence for conjugating with DSPE-PEG-Mal. IFPT - immunogenic fused PCSK9-tetanus.

sized and high performance liquid chromatography (HPLC)-purified by ChinaPeptides Co., Ltd. (Shanghai, China). The already designed IFPT construct [31] contains a PCSK9 peptide, as a B cell epitope inspired from the AFFiRiS group [27, 32], and a T-helper cell epitope belonging to tetanus toxin included as a standard adjuvant carrier [33] (Table I). To link the IFPT epitope on the surface of liposome nanoparticles, it was bound to DSPEPEG-Mal (1,2-distearoyl-sn-glycero-3-phosphoethanolamine- $N$-[maleimide(PEG)-2000]) lipid (Lipoid $\mathrm{GmbH}$, Germany) via an N-terminal cysteine residue inserted in IFPT peptide (Figure 1).

\section{Manufacturing of DSPE-PEG-IFPT micelles}

DSPE-PEG-Maleimide lipid was used to link the IFPT peptides on the surface of liposome nanoparticles as an adjuvant delivery system. The $\mathrm{N}$-terminal cysteine residue of the IFPT peptide provides a thiol group that reacts with the pyrrole group maleimide and generates a thioether bond, whereby the peptide covalently conjugates with the DSPE-PEG-Maleimide linker. The IFPT peptide and DSPE-PEG-Mal at the molar ratios of $1.2: 1$, respectively, were dissolved in DMSO/chloroform solution at the volume ratio of $1: 1$, and then gently stirred at RT for $48 \mathrm{~h}$. The linkage was confirmed using the TLC (thin layer chromatography) method with the mobile phase containing chloroform, methanol, and water at the volume ratio of $90: 18: 2$. After that, the DMSO/chloroform solution was dried by a rotary evaporator and freeze-drying followed by hydration with sterile deionized water $\left(\mathrm{pH} \mathrm{7.2)}\right.$ at $30^{\circ} \mathrm{C}$ to prepare DSPEPEG-IFPT micelles. The efficiency of the linkage in the constructed micelles was estimated by HPLC analysis. The true value of the linked micelles was measured using efficiency of linkage and content of total lipid determined by the Bartlett phosphateassay method [34].

\section{HPLC analysis of linkage efficiency}

The efficinecy of linkage between the IFPT peptide and the DSPE-PEG-Mal linker was indirectly estimated by HPLC quantification of the free peptide content of the prepared DSPE-PEG-IFPT micelles. The HPLC apparatus was equipped with a Smart line HPLC Pump 1000, a PDA Detector 2800 (set at $220 \mathrm{~nm}$ ), and a Degasser5000, all from Knauer (Berlin, Germany). Each sample (20 $\mu \mathrm{l})$ was injected through a Smart line auto sampler and data were obtained and processed with

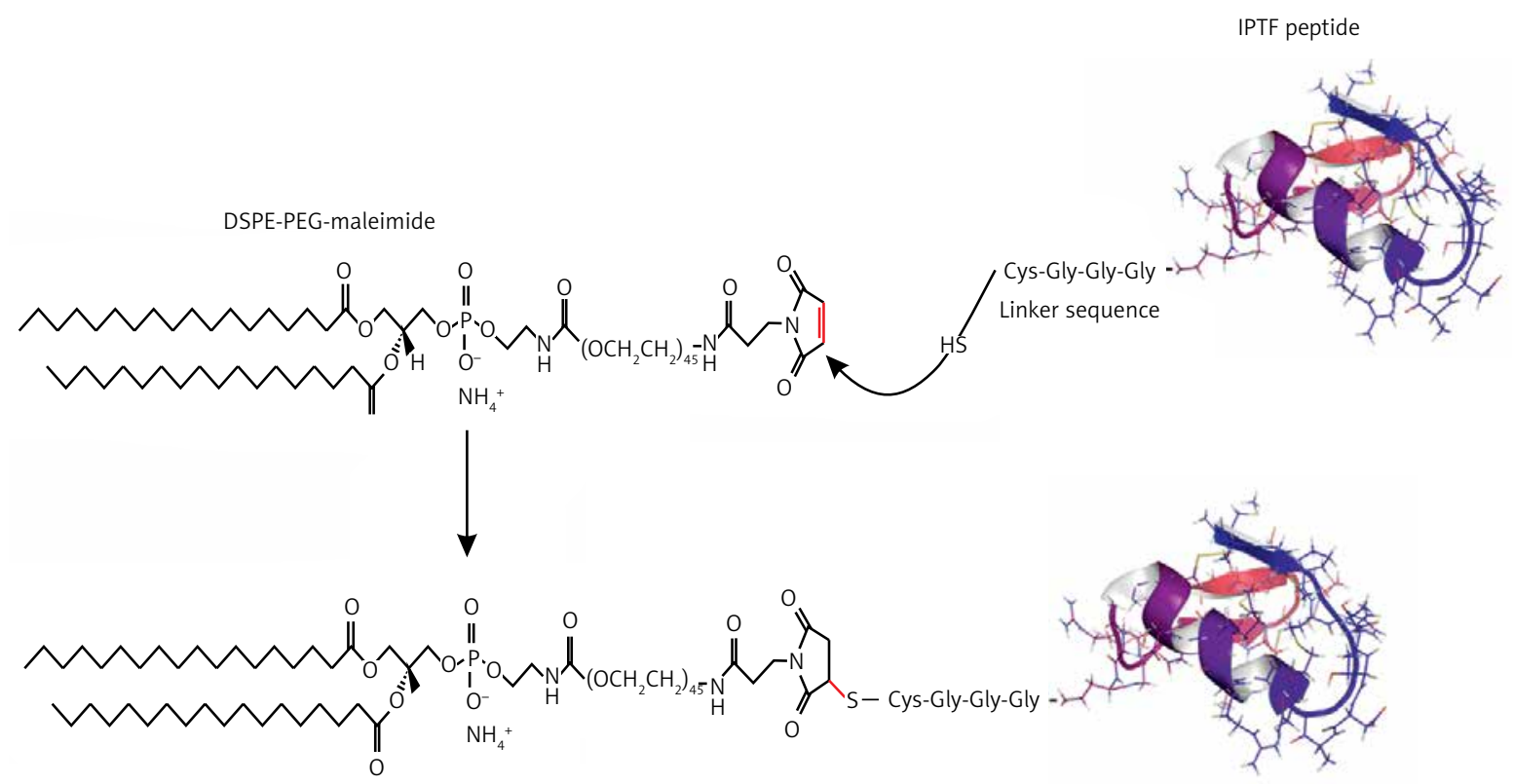

Figure 1. Schematic view of linking between peptide and DSPE-PEG-maleimide 
ChromGate software (version 3.3.1) from Knauer (Berlin, Germany). Chromatographic separation was performed on a $\mathrm{C} 18$ reverse-phase column, $4.6 \mathrm{~mm} \times 25 \mathrm{~cm}$ (Shimadzu, Japan), using an isocratic mobile phase of $(0.1 \%$ TFA in water $) /(0.1 \%$ TFA in acetonitrile) at gradient ratios of $55 / 45$ to $45 / 55$ in $10 \mathrm{~min}$, at a flow rate of $1 \mathrm{ml} / \mathrm{min}$.

The IFPT peptide with HPLC purity $>95 \%$ was used as a standard solution. The free peptide peak within the chromatogram of the micelle sample was identified and assigned based on the retention time $(2.2 \mathrm{~min})$ of the standard solution, followed by sample spiking.

To quantify the free peptide content of the micelle sample, a calibration curve was constructed by injecting standard solution at five concentrations $(50-500 \mu \mathrm{g} / \mathrm{ml})$, which was linear with a correlation coefficient $\left(r^{2}\right)$ of 0.9954 . Using linear regression analysis of the calibration curve appearing in the standard chromatogram, the free peptide content of the micelle sample was measured. Linkage efficiency in the constructed DSPE-PEG-IFPT micelles was calculated by subtracting the free peptide amount within the micelles quantified by HPLC from the amount of the IFPT peptide initially added.

\section{Construction and cahraacterization}

\section{of nanoliposomal IFPT vaccine}

Nanoliposomes were used as a delivery adjuvant to enhance immunogenicity of the peptide. Since many IFPT peptides can be conjugated to the surface of liposome nanoparticles, we propose that IFPT-linked nanoliposomes can elicit a high-titer antibody against self-antigen PCSK9, maybe through elevating peptide valency. To attach the IFPT peptide on the nanoliposome surface, the post-insertion approach was performed, in which the prepared DSPE-PEG-IFPT micelles (100 $\mu \mathrm{g}$, based on the linked peptide) and liposome nanoparticles $(1 \mathrm{ml})$ were mixed and then gentely shaken at $45^{\circ} \mathrm{C}$ for $3 \mathrm{~h}$. The micelles were inserted in the nanoliposome bilayer via the DSPE phospholipid moiety, and exposed IFPT peptides on the nanoliposome surface through the PEG chains. Particle size, surface charge and PDI of the prepared nanoliposomal IFPT particles were evaluated using DLS technique on a Zetasizer (Nano-ZS, Malvern, UK) at RT. The IFPT-conjugated nanoliposomes were adsorbed to $0.4 \%$ Alum adjuvant
(Sigma-Aldrich) at a $1: 1(\mathrm{v}: \mathrm{v})$ ratio in a total volume of $400 \mu \mathrm{l}$ and stored at $4^{\circ} \mathrm{C}$ under argon. Prior to injection, the nanoliposomal IFPT plus Alum vaccine, hereafter called L-IFPTA ${ }^{+}$, was brought to RT and carefully mixed.

\section{Animal and cell line}

A total of 15 female BALB/c mice (4-6 weeks old) were purchased from Pasteur Institute of Tehran, Iran and fed with ad libitum access to purified water and a commercial stock diet. All mice were housed in a pathogen-free animal house at a temperature of $22 \pm 1^{\circ} \mathrm{C}$ with a $12: 12 \mathrm{~h}$ light : dark cycle and maintained under a relative humidity of $50 \pm 10 \%$. Animal care was performed in accordance with welfare guidelines established by the Institutional Ethical Committee and Research Advisory Committee of Mashhad University of Medical Sciences. At the end of the experiment all animals were euthanized by $\mathrm{CO}_{2}$ inhalation.

The CT26 colon carcinoma cell line was provided from Pasteur Institute of Tehran, Iran and cultured in RPMI-1640 medium containing 10\% FBS and supplemented with penicillin $(100 \mathrm{IU} / \mathrm{ml}) / \mathrm{streptomycin}$ $(100 \mathrm{mg} / \mathrm{ml})$. The cells were incubated at $37^{\circ} \mathrm{C}$ with a $5 \% \mathrm{CO}_{2} / 95 \%$ air humidified atmosphere.

\section{Vaccination schedule}

Following one week of taming prior to the experimental procedures, the mice were randomly arranged into two groups: a vaccine group $(n=5)$ and an untreated group $(n=10)$ group. The vaccination was subcutaneously primed at week 0 (WO) and followed by three boosters (W2, W4, and W6), in bi-weekly intervals (Figure 2), while untreated mice simultaneously received phosphate-buffered saline (PBS). Tail vein bleeding was performed two weeks after each vaccination for the titration of plasma anti-PCSK9 antibody.

\section{Evaluation of plasma anti-PCSK9 antibody}

To measure the titer of anti-PCSK9 antibodies, plasma samples were collected and assayed by the ELISA method. Briefly, PCSK9 peptide at the concentration of $5 \mu \mathrm{g} / \mathrm{ml}$ in $0.1 \mathrm{M} \mathrm{NaHCO}_{3}$ (pH 9.2-9.4) was coated overnight in a 96-well Nunc-MaxiSorp plate. Free binding sites were then blocked by incubation with blocking buffer ( $1 \times$ PBS, $1 \%$ BSA) for

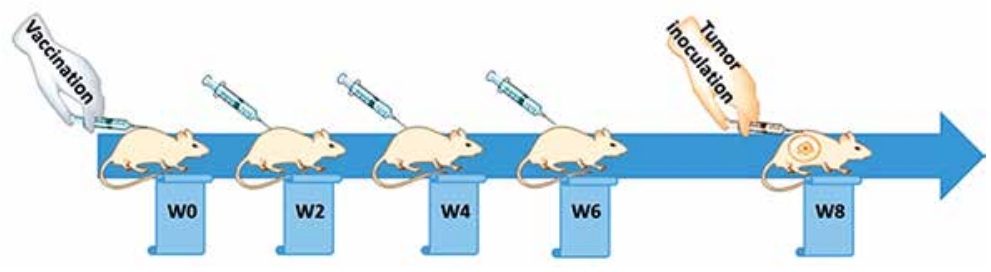

Figure 2. Study design 
$1 \mathrm{~h}$ at $37^{\circ} \mathrm{C}$. Diluted plasma $(1: 400$ in $1 \times \mathrm{PBS} / 0.1 \%$ $\mathrm{BSA} / 0.05 \%$ Tween-20) was added, serially diluted $1: 4$, and incubated for $1 \mathrm{~h}$ at $37^{\circ} \mathrm{C}$. Each ELISA plate contained a standard antibody as an internal control. For detection, biotinylated anti-mouse IgG $(\mathrm{H}+\mathrm{L})($ Sigma-Aldrich; 1 : 1000) in $1 \times \mathrm{PBS} / 0.1 \%$ BSA/0.1\% Tween-20 was applied and incubated for $1 \mathrm{~h}$ at $37^{\circ} \mathrm{C}$. Then, horseradish peroxidase coupled to streptavidin (Roche) was added $\left(30 \mathrm{~min}, 37^{\circ} \mathrm{C}\right.$ ) followed by the addition of the substrate $2,2^{\prime}$-azinobis [3-ethylbenzothiazoline-6-sulfonic acid]diammonium salt (ABTS) (Sigma-Aldrich) (15 min, $\mathrm{RT}$ ). The optical density (OD) at $450 \mathrm{~nm}$ was measured with a Microwell plate reader (Sunrise, Tecan, Switzerland) and the titers were defined as the dilution factor referring to $50 \%$ of the maximal optical density $\left(O D_{\max } / 2\right)$. The mean titers $\pm S D$ of all animals per group are presented.

\section{Plasma PCSK9 quantification}

Plasma PCSK9 concentration in the vaccinated mice was measured by CircuLex rat PCSK9 ELISA (CircuLex, Cy-8078, MBL, Woburn, MA) according to the manufacturer's instructions. Briefly, $100 \mu \mathrm{l}$ of the diluted $1: 100$ plasma samples was added to a 96-well microplate and incubated for $1 \mathrm{~h}$ at RT. A HRP-conjugated anti-PCSK9 antibody was added for $1 \mathrm{~h}$ followed by the substrate reagent and stop solution, all at RT. Optical density was detected at $450 \mathrm{~nm}$ with a Microwell plate reader (Sunrise, Tecan, Switzerland). A standard curve provided by the supplier was defined to measure PCSK9 concentration.

\section{PCSK9 inhibition analysis}

To assay inhibition of mice PCSK9 by vaccine-induced antibodies, interaction of induced antibodies with PCSK9 was evaluated. For this purpose, the same kit CircuLex rat PCSK9 ELISA was used, but for HRP-conjugated anti-PCSK9 antibody, detection was performed with HRP-conjugated anti-mouse IgG $(\mathrm{H}+\mathrm{L})$ (Sigma Aldrich; dilution 1 : 5000) incubated for $1 \mathrm{~h}$ at RT, followed by the substrate reagent and stop solution provided by the supplier. The OD was detected at $450 \mathrm{~nm}$ with the Microwell plate reader.

\section{In vitro evaluation of PCSK9-LDLR binding}

CircuLex PCSK9-LDLR in vitro binding assay kit (Circulex, Cy-8150, MBL, Woburn, MA) was used to evaluate the ability of vaccine-induced antibodies to inhibit the PCSK9-LDLR interaction in vitro. Briefly, $100 \mu \mathrm{l}$ of vehicle control or the plasma samples of vaccinated mice was added to a 96-well microplate pre-coated with a recombinant LDLR-AB domain, which contains a binding site for PCSK9. Immediately after that, the reac- tion was initiated by adding a "His-tagged PCSK9 wiled type" solution incubated for $2 \mathrm{~h}$ followed by adding a biotinylated anti-His-tag monoclonal antibody for $1 \mathrm{~h}$ at RT. Then, HRP-conjugated streptavidin was coated for $1 \mathrm{~h}$ at RT followed by the substrate reagent and stop solution. In this method, the higher amount of PCSK9-LDLR interaction is associated with higher ELISA OD, in which in the presence of anti-PCSK9 antibody this interaction is inhibited and consequently ELISA OD is decreased. A dose-response curve with appropriate serial dilutions of "His-tagged PCSK9 wild type" solution was drawn to measure the accurate inhibition percentage of test samples.

\section{Evaluation of in vivo anti-tumor efficacy}

Two weeks after the last booster, the vaccinated and unvaccinated BALB/c mice were subcutaneously inoculated with CT26 colon carcinoma cells $\left(5 \times 10^{5} / 50 \mu \mathrm{l}\right.$ PBS/mouse) into the right flank at day zero. Tumor growth was monitored in 3-day intervals by calculating the tumor volume after measuring three orthogonal diameters with calipers according to the formula: tumor volume $\left(\mathrm{mm}^{3}\right)=($ length $\times$ height $\times$ width $) \times 0.52$. After the tumor mass became palpable (approximately $10 \mathrm{~mm}^{3}$ ) at day 10 , the mice were randomly divided into three groups (5 mice/group) and subjected to different treatment protocols: (1) the vaccine group comprising vaccinated tumor-bearing mice that received a single tail vein injection of PBS, (2) the Doxil (liposomal doxorubicin) (positive control) group comprising unvaccinated tumor-bearing mice that received a single tail vein injection of Doxil (15 mg/kg), and (3) the control (PBS) group comprising unvaccinated tumor-bearing mice that received a single tail vein injection of PBS.

To evaluate therapeutic efficacy, mouse body weight, tumor size, general health, and survival were monitored every other day for 50 days. Animals' euthanasia ( $\mathrm{CO}_{2}$ inhalation) was performed on those with 4T1 tumor due to the following ethical considerations: body weight loss $>20 \%$ of initial mass, tumor volume greater than $2.0 \mathrm{~cm}$ in one dimension, or mice became sick and unable ambulate to reach food/water $[35,36]$. For each mouse, the time to reach tumor volume above $1000 \mathrm{~mm}^{3}$ or the time to reach the end point (TTE) as a response variable was calculated from the equation of the line obtained by exponential regression of the tumor growth curve. For each group, the percent of tumor growth delay (\%TGD) was determined by calculating the difference between the average TTE of the treatment group (T) and the average TTE of the control group (C), (\%TGD = $[(T-C) / C] \times 100)$ [37]. For each treatment group, the percent of increased life span (\%ILS) was measured based on the following formula: [(average 
survival time of treatment group / average survival time of control group $\times 100)-100]$ [38].

\section{Statistical analysis}

Statistical analysis was performed using SPSS Statistics version 20 and GraphPad Prism version 7.04 software. Survival data were analyzed using the log-rank (Mantel-Cox) test. Other comparisons were done using one-way ANOVA and the Tukey post-hoc multiple comparison test. Values were expressed as mean \pm SD or median for normally and non-normally distributed data, respectively. Results with $p<0.05$ were considered as statistically significant.

\section{Results}

\section{Physical properties}

Characterization of liposome nanoparticles showed a size of $145 \mathrm{~nm}$ and $180 \mathrm{~nm}$ for free and IFPT-linked nanoliposomes, respectively. Other characteristics including PDI and zeta potential are detailed in Table II.

\section{Analysis of DSPE-PEG-IFPT micelles}

Attachment of the IFPT peptides to the DSPEPEG-Mal linker was confirmed qualitatively and quantitatively using TLC (Figure 3) and HPLC (Figure 4) methods, yielding an attachment yield of $96 \%$ according to HPLC.

\section{L-IFPTA $^{+}$vaccine-induced PCSK9 antibody response in $B A L B / C$ mice}

The L-IFPTA+ vaccine induced a strong IgG response against PCSK9 peptide in BALB/C mice upon 4 immunizations in biweekly intervals. Notably, long-term analysis revealed that anti-PCSK9 antibody titer peaked at week 8 and remained constant up to week 10, and then revealed a decreasing trend (Figure $5 \mathrm{~A}$ ).

\section{Targeting of plasma PCSK9 by vaccine- induced antibodies}

Vaccine-induced PCSK9 antibodies targeted plasma PCSK9 in vaccinated mice in a specific manner. Plasma PCSK9 levels were $28 \pm 6 \mathrm{ng} / \mathrm{ml}$

Table II. Physical properties of nanoliposomal formulations

\begin{tabular}{|lccc|}
\hline Formulation & $\begin{array}{c}\text { Z-average }[\mathrm{nm}] \\
\text { Mean } \pm \text { SD }(n=3)\end{array}$ & $\begin{array}{c}\text { Zeta potential [mV] } \\
\text { Mean } \pm \text { SD }(n=3)\end{array}$ & $\begin{array}{c}\text { PDI } \\
\text { Mean } \pm \text { SD }(n=3)\end{array}$ \\
\hline Empty nanoliposome & $145 \pm 7.5$ & $-38 \pm 3$ & $0.07 \pm 0.01$ \\
\hline IFPT-linked nanoliposome & $180 \pm 5.5$ & $-31 \pm 2$ & $0.1 \pm 0.05$ \\
\hline
\end{tabular}

PDI - polydispersity index.

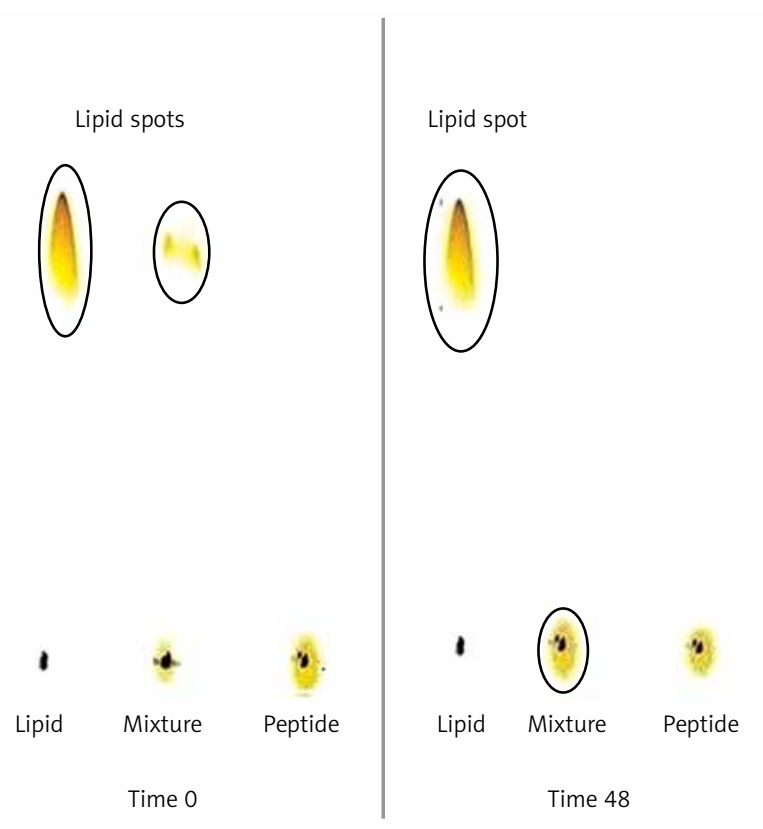

Figure 3. Assessment of conjugation between DSPE-PEG-Mal and the IFPT peptide at time zero and $48 \mathrm{~h}$ after start of reaction. Lipid (DSPE-PEG-Mal) is dissolved in the mobile phase and ascends to the top of the TLC plate (spots in the top of the left and middle lines) but peptide is bound to the silica and remains in the spotting point (the middle line). After $48 \mathrm{~h}$, lipid bound to peptide and stayed at the point of spotting, and therefore the lipid spot on the top of the reaction mixture line disappeared, indicating the conjugation of the IFPT peptide and DSPE-PEG-Mal linker 


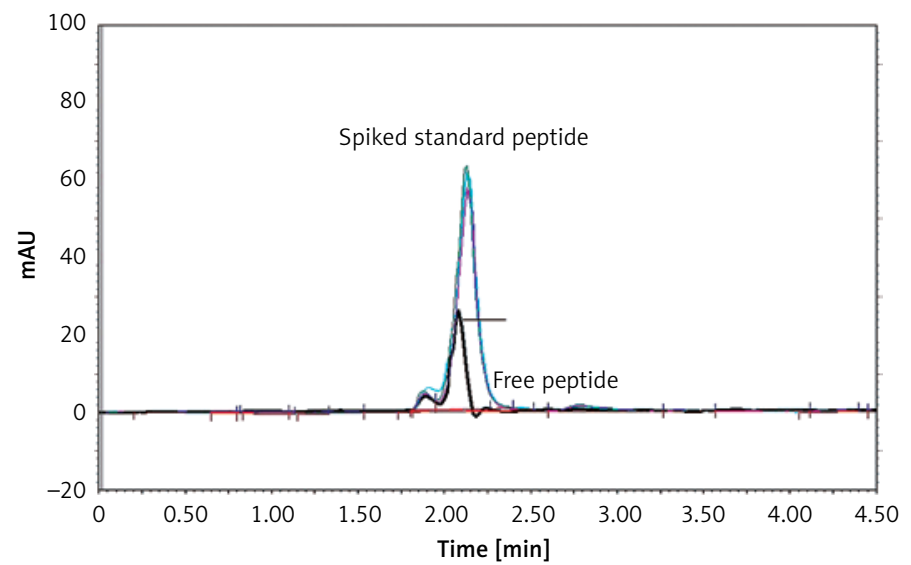

Figure 4. HPLC chromatogram of DSPE-PEG-IFPT micelles and reference standard IFPT peptide. The retention time of the reference standard was observed at $2.2 \mathrm{~min}$ and it was found to be the same with free peptide present in the micelle sample

A

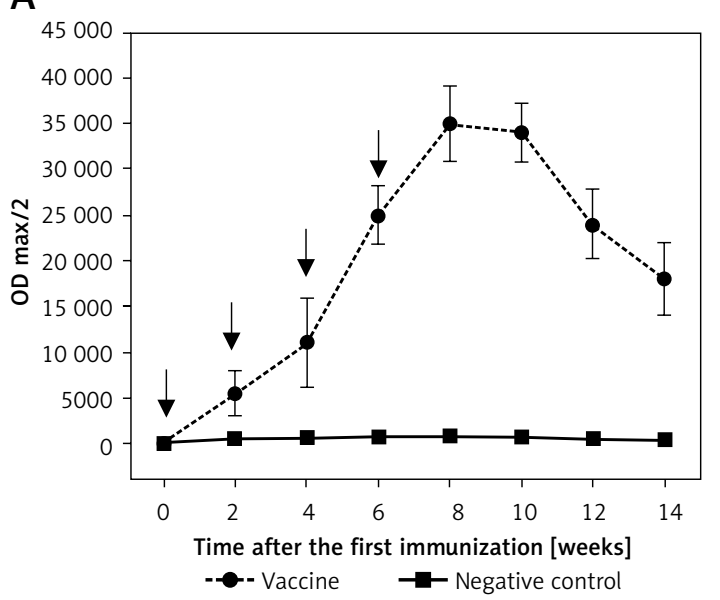

C

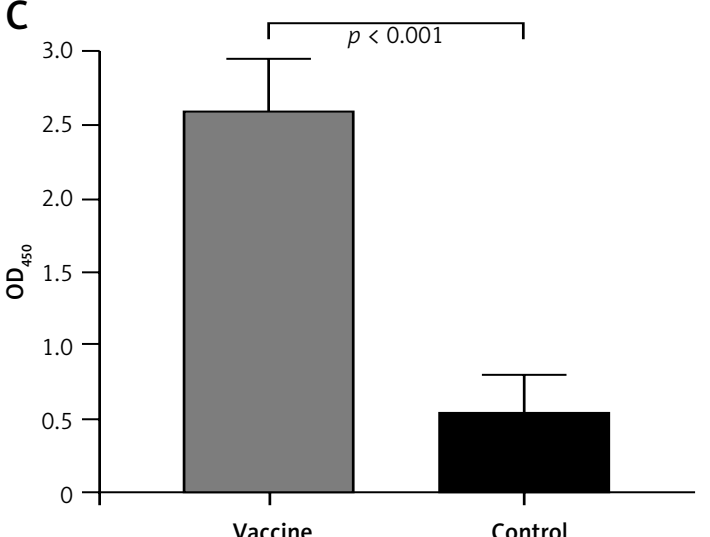

B

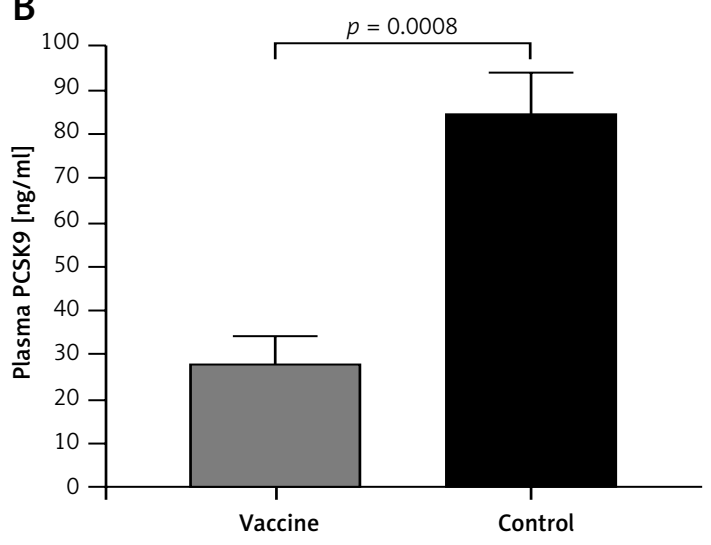

D

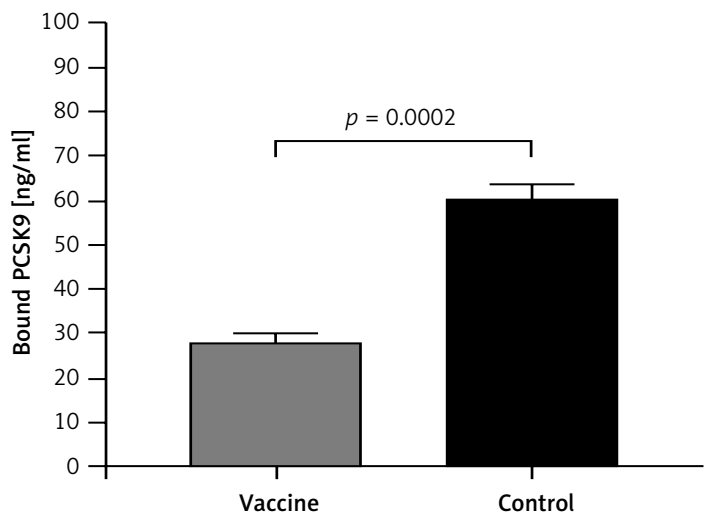

Figure 5. AntiPCSK9 vaccine efficacy. A - L-IFPTA ${ }^{+}$vaccine could induce anti-PCSK9 antibody titers (ODmax/2) upon 4 immunizations in a bi-weekly interval (indicated by arrows). Antibody titers were assayed over 14 weeks after prime immunization. B - Concentrations of plasma PCSK9 in the vaccine and control group were $28 \pm 6 \mathrm{ng} / \mathrm{ml}$ and $85 \pm 9 \mathrm{ng} / \mathrm{ml}$, respectively. C - Direct detection of antibodies bound to plasma PCSK9 in the plasma samples from vaccinated and control mice. Increased $\mathrm{OD}_{450}$ is indicative for vaccine-induced anti-PCSK9 antibodies which directly target PCSK9. D - In vitro PCSK9/LDLR binding assay. Plasma sample of vaccine group could decrease PCSK9 binding to LDLR by $53 \%$, when compared with plasma sample of control group. Values are expressed as means $\pm \mathrm{SD}(n=3$ replicates of the pooled samples of 5 mice). Significance compared to control values was analyzed by unpaired 2-tailed Student's $t$-test 
and $85 \pm 9 \mathrm{ng} / \mathrm{ml}$ in the vaccinated and control groups, respectively (Figure 5 B). Plasma PCSK9 concentrations were decreased by $67 \%(-57 \pm 6 \mathrm{ng} /$ $\mathrm{ml}, p=0.0008)$ in the vaccinated mice as compared to control mice. The specificity of PCSK9 targeting by vaccine-induced antibodies was tested using an ELISA assay as described above and in our previous report [31]. A significantly higher $\mathrm{OD}_{450}$ signal was detected from the plasma of vaccinated mice, suggesting that vaccine-induced PCSK9 antibodies were able to directly bind to PCSK9 (Figure $5 \mathrm{C}$ ).

\section{PCSK9-LDLR interaction blockade by vaccine-induced PCSK9 antibodies}

Using the above-mentioned in vitro assay, PCSK9 antibodies were found to inhibit the interaction between PCSK9 and LDLR. Binding of PCSK9 to LDLR was reduced by $53 \%$ in the presence of plasma obtained from L-IFPTA ${ }^{+}$-vaccinated mice, as compared to control mice (Figure $5 \mathrm{D}$ ).

\section{Efficacy of liposomal-antiPCSK9 in CT24 colon carcinoma model treatment}

To assay the effect of PSCK9 inhibition on breast cancer progression, a CT26 colon carcinoma model was developed in BALB/c mice previously immunized with liposomal antiPCSK9 vaccine. The protective effect of the vaccine on tumor-bearing mice was evaluated by monitoring body weight alterations, tumor growth rate in terms of mean tumor size $\left(\mathrm{mm}^{3}\right)$, and survival.

The weight monitoring curve showed that body mass was significantly reduced in the vaccinated mice after 25 days of tumor induction. As compared with the control group, vaccinated mice showed a significantly higher body weight loss from 25 days after tumor induction $(p<0.05)$ (Figure 6 A).
Analysis of the integrated areas under the body weight curve $\left(A \cup C_{\text {body weight }}\right)$ over 50 days showed that the body weight of vaccinated mice was decreased by $7.7 \%$ in comparison with the control mice, while there was no significant difference between vaccine and Doxil groups (Figure 6 B). To determine endpoint therapeutic modalities of antiPCSK9 vaccine in tumor-bearing BALB/c mice, time to reach endpoint (TTE) and percentage of tumor growth delay (\%TGD), as well as median survival time (MST) and increase in life span (ILS), were measured (Table III). Monitoring the tumor size (Figure 7 A) revealed that three out of five vaccinated mice remained tumor-free, and tumor growth in the remaining two vaccinated mice was significantly lower than that in Doxil and the control mice. Data of the integrated areas under the tumor volume curve $\left(A \cup C_{\text {tumor volume }}\right)$ over 50 days demonstrated that tumor volume was $77 \%$ and $87.7 \%$ lower $(p<0.0001)$ in the vaccinated mice than in the Doxil and the control mice, respectively (Figure 7 B). TTE of the vaccine group (47 \pm 11 days) was slightly but not significantly higher than that of the Doxil ( $46 \pm 2.6$ days) and control (43 \pm 12 days) groups. TGD as a widely accepted method for qualifying in-situ assessment of tumor treatment quality showed that the tumor growth rate in the vaccine and Doxil group was reduced by $9.3 \%$ and $7.3 \%$, respectively, compared with the control group. Kaplan-Meier curves (Figure 8) showed that the vaccinated mice survived slightly but not significantly longer than the Doxil and the control mice ( $p<0.05$, log-rank test). The MST of the vaccine, Doxil and control group was 51, 45 , and 41 days, respectively. Analysis of the ILS revealed that the vaccinated mice's life was prolonged by $24.4 \%$ as compared with the control mice, while it was increased by $9.8 \%$ in the Doxil group (Table III).
A

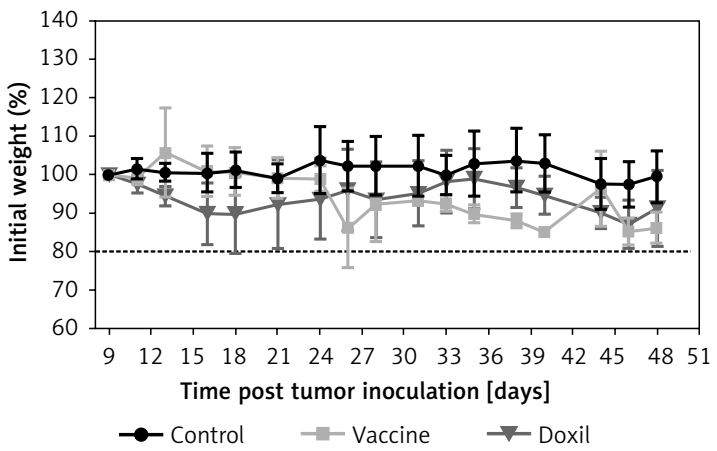

B

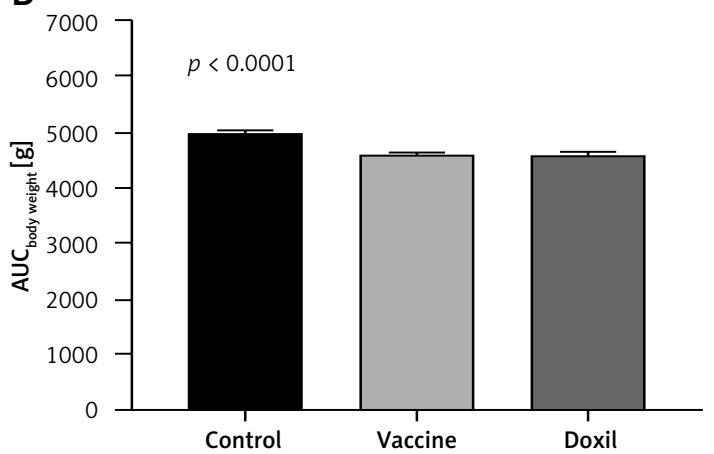

Figure 6. The weight monitoring curve (A) shows point-by-point changes of the body weight during 50 days in the control, vaccine, and Doxil group. The integrated areas under the body weight curve $\left(\mathrm{AUC}_{\text {body weight }}\right)$ over 50 days (B) demonstrates overall weight changes. Data with $p<0.05$ were considered as statistically significant. The body weight loss was significantly $(p<0.0001)$ higher in the vaccine and Doxil groups than in the control group. Animal body weight was measured every 3 days

Data are presented as the mean $\pm S D(n=5)$. 
Table III. Therapeutic efficacy data of different treatments in BALB/c mice bearing CT26 colon carcinoma

\begin{tabular}{|lcccc|}
\hline Groups & $\begin{array}{r}\text { TTE [days] } \\
\text { Mean } \pm \text { SD }\end{array}$ & TGD (\%) & MST [days] & ILS (\%) \\
\hline Control & $43 \pm 12$ & - & 41 & - \\
\hline Vaccine & $47 \pm 11$ & 9.3 & 51 & 24.4 \\
\hline Doxil & $46 \pm 3$ & 7.3 & 45 & 9.8 \\
\hline
\end{tabular}

TTE - time to reach end point, TGD - tumor growth delay, MST - median survival time, ILS - increase life span.

A

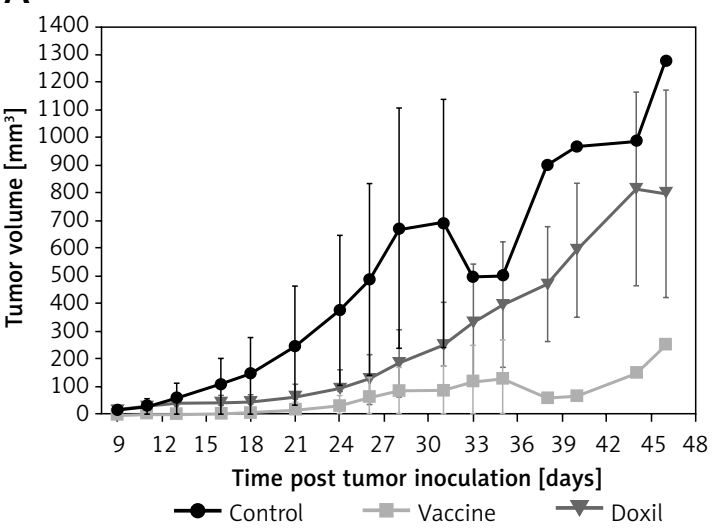

C

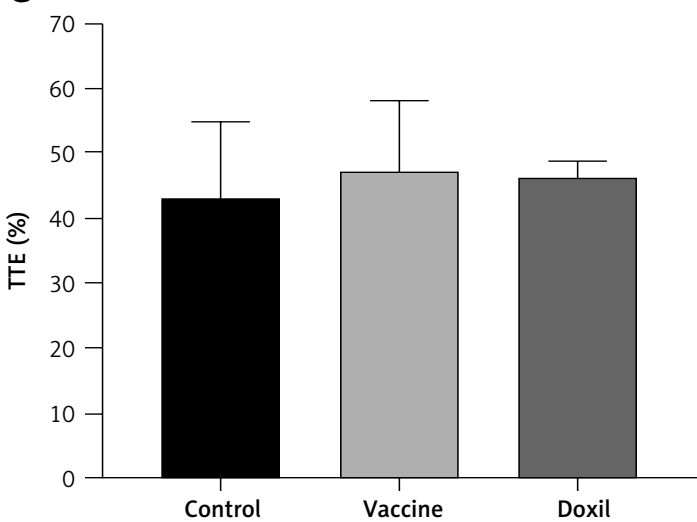

Discussion

Recent Mendelian randomization studies analyzing the correlation of PCSK9 polymorphisms with cancer occurrence have shown contradictory results with respect to the association of LDL-rising/lowering PCSK9 mutations and risk of cancer [24-26]. However, there have been no data on the effect of PCSK9 inhibition on cancer progression. Dyslipidemia, particularly an increased plasma level of LDL-C, has been reported to be significantly correlated with a higher risk and incidence of colon cancer [9-12]. However, the effects of pharmacological LDL-C lowering on cancer has remained unclear. In this study, we explored whether PCSK9 inhibition has any positive, negative or neutral effect on the endpoints of colorectal cancer. Interestingly, our results showed that the nanoliposomal anti-PCSK9 (L-IFPTA ${ }^{+}$) vaccine, as a PCSK9
B

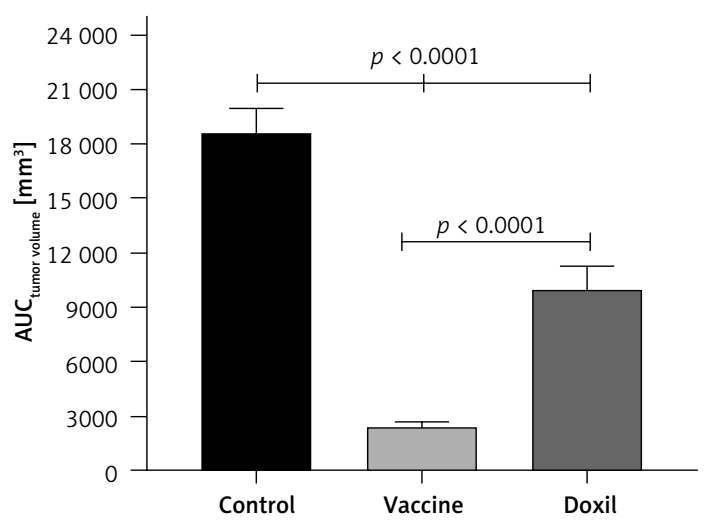

Figure 7. The tumor growth curve (A) shows the increase of tumor size during 50 days in the control, vaccine, and Doxil groups. Tumor volume $\left(\mathrm{mm}^{3}\right)$ was measured every 3 days. The integrated areas under the tumor volume curve ( $A \cup C_{\text {tumor volume }}$ ) over 50 days (B) demonstrates overall increase of tumor size. Measuring the time to reach endpoint (TTE) or the time to reach tumor volume above $1000 \mathrm{~mm}^{3}$ (C) showed no significant difference between studied groups. Data with $p<0.05$ were considered as statistically significant

Data are presented as the mean $\pm S D(n=5)$.

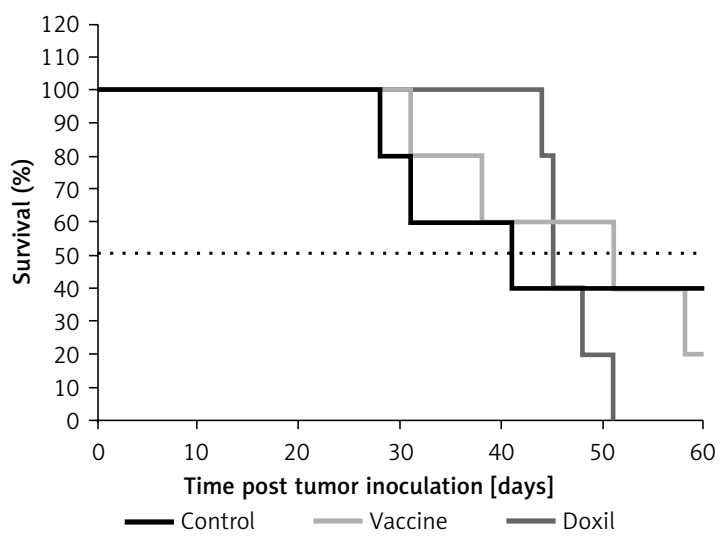

Figure 8. Kaplan-Meier curves exhibit the survival rate of the control, vaccine, and Doxil groups. There was no significant difference between the compared groups

Data are presented as the mean $\pm S D(n=5) . P<0.05$ was considered as the level of statistical significance. 
inhibitor, could moderately inhibit tumor development in mice bearing CT26 colon carcinoma.

L-IFPTA $^{+}$vaccine induced generation of functional anti-PCSK9 antibodies (Figure $5 \mathrm{~A}$ ) that reduce plasma level and activity of PCSK9 (Figures 5 B, D) through specific and direct targeting of PCSK9 (Figure $5 \mathrm{C}$ ) in BALB/c mice. The vaccine-induced anti-PCSK9 antibodies were found to significantly reduce tumor growth (Figure 7 ), partially increase MST (Figure 8), and prolong lifespan (Table III) in the vaccinated mice bearing colorectal cancer, when compared with Doxil and control groups.

The moderate anti-tumor effect of the tested anti-PCSK9 vaccine is underpinned by the recent Mendelian randomization study which revealed that LDL-lowering mutations of PCSK9 mimicking PCSK9 inhibitors were correlated with a lower risk of breast cancer occurrence, while PCSK9 with LDL-raising mutations has a causal correlation with a higher risk of breast cancer [26]. It is further supported by another human study analyzing a phenome-wide association of LDL-lowering genetic variants in the PCSK9 locus mimicking PCSK9 inhibitors, showing that these PCSK9 variants do not increase the risk of cancer [39]. However, a prospective, population-based cohort study showed that there is no association between LDL-lowering PCSK9 polymorphisms and the risk of cancer [25].

In conclusion, the present data demonstrated that PCSK9 inhibition is potentially safe, and might moderately inhibit tumor progression in an experimental model of colon cancer. These findings can support the potential safety of PCSK9 inhibition in cancer, though further preclinical and clinical trials taking into account different PCSK9 inhibition approaches, longer term follow-up durations and other cancer models are necessary to confirm the safety and possible efficacy of PCSK9 inhibition in malignant conditions.

\section{Acknowledgments}

We would also like to thank Mrs. Azam Abbasi and Mrs. Zahra Saberi for their valuable technical assistance.

We are thankful for the financial support from the Mashhad University of Medical Sciences, Mashhad, Iran (grant number: 941726) and the National Institute for Medical Research Development (NIMAD), Tehran, Iran (Grant no: 963401).

\section{Conflict of interest}

The authors declare no conflict of interest.

\section{References}

1. Bird CL, Ingles SA, Frankl HD, et al. Serum lipids and adenomas of the left colon and rectum. Cancer Epidemiol Prev Biomarkers 1996; 5: 607-12.
2. Kim BC, Shin A, Hong CW, et al. Association of colorectal adenoma with components of metabolic syndrome. Cancer Causes Control 2012; 23: 727-35.

3. van Duijnhoven FJ, Bueno-De-Mesquita HB, Calligaro $\mathrm{M}$, et al. Blood lipid and lipoprotein concentrations and colorectal cancer risk in the European prospective investigation into cancer and nutrition. Gut 2011; 60: 1094-102.

4. Kim DH, Kim YE. Association of serum lipids and glucose with the risk of colorectal adenomatous polyp in men: a case-control study in Korea. J Korean Med Sci 2000; 15: 690-5.

5. Ahmed RL, Schmitz KH, Anderson KE, et al. The metabolic syndrome and risk of incident colorectal cancer. Cancer 2006; 107: 28-36.

6. Zhang X, Zhao XW, Liu DB, et al. Lipid levels in serum and cancerous tissues of colorectal cancer patients. World J Gastroenterol 2014; 20: 8646-52.

7. Coppola JA, Shrubsole MJ, Cai Q, et al. Plasma lipid levels and colorectal adenoma risk. Cancer Causes Control 2015; 26: 635-43.

8. Kim JH, Lim YJ, Kim YH, et al. Is metabolic syndrome a risk factor for colorectal adenoma? Cancer Epidemiol Prev Biomarkers 2007; 16: 1543-6.

9. Tian Y, Wang K, Li J, et al. The association between serum lipids and colorectal neoplasm: a systemic review and meta-analysis. Public Health Nutr 2015; 18: 3355-70.

10. Passarelli MN, Newcomb PA. Blood lipid concentrations and colorectal adenomas: a systematic review and meta-analysis of colonoscopy studies in Asia, 2000-2014. Am J Epidemiol 2016; 183: 691-700.

11. Yang MH, Rampal S, Sung J, et al. The association of serum lipids with colorectal adenomas. Am J Gastroenterol 2013; 108: 833-41.

12. Bayerdorffer E, Mannes GA, Richter WO, et al. Decreased high-density lipoprotein cholesterol and increased low-density cholesterol levels in patients with colorectal adenomas. Ann Intern Med 1993; 118: 481-7.

13. Zhang DW, Lagace TA, Garuti R, et al. Binding of proprotein convertase subtilisin/kexin type 9 to epidermal growth factor-like repeat A of low density lipoprotein receptor decreases receptor recycling and increases degradation. J Biol Chem 2007; 282: 18602-12.

14. Kwon HJ, Lagace TA, McNutt MC, et al. Molecular basis for LDL receptor recognition by PCSK9. Proc Natl Acad Sci 2008; 105: 1820-5.

15. Horton JD, Cohen JC, Hobbs HH. PCSK9: a convertase that coordinates LDL catabolism. J Lipid Res 2009; 50: S172-7.

16. Food U, Administration D. FDA approves Repatha to treat certain patients with high cholesterol. Accessed at: https://www.fda.gov/NewsEvents/Newsroom/PressAnnouncements/UCM460082 F

17. Sabatine MS, Giugliano RP, Wiviott SD, et al. Efficacy and safety of evolocumab in reducing lipids and cardiovascular events. N Engl J Med 2015; 372: 1500-9.

18. Sahebkar A, Watts GF. New therapies targeting apoB metabolism for high-risk patients with inherited dyslipidaemias: what can the clinician expect? Cardiovasc Drugs Ther 2013; 27: 559-67.

19. Dragan S, Serban MC, Banach M. Proprotein convertase subtilisin/kexin 9 inhibitors: an emerging lipid-lowering therapy? J Cardiovasc Pharmacol Ther 2015; 20: 157-68.

20. Soran H, Adam S, Mohammad JB, et al. Hypercholesterolaemia - practical information for non-specialists. Arch Med Sci 2018; 14: 1-21.

21. Jaworski K, Jankowski P, Kosior DA. PCSK9 inhibitors from discovery of a single mutation to a groundbreak- 
ing therapy of lipid disorders in one decade. Arch Med Sci 2017; 13: 914-29.

22. Banach M, Penson PE. What have we learned about lipids and cardiovascular risk from PCSK9 inhibitor outcome trials: ODYSSEY and FOURIER? Cardiovasc Res 2019; 115: e26-31.

23. Macchi C, Banach M, Corsini A, et al. Changes in circulating pro-protein convertase subtilisin/kexin type 9 levels - experimental and clinical approaches with lipid-lowering agents. Eur J Prev Cardiol 2019: 2047487319831500 doi: $10.1177 / 2047487319831500$.

24. Benn M, Tybjærg-Hansen A, Stender S, et al. Low-density lipoprotein cholesterol and the risk of cancer: a mendelian randomization study. J Natl Cancer Instit 2011; 103: 508-19.

25. Folsom AR, Peacock JM, Boerwinkle E. Sequence variation in proprotein convertase subtilisin/kexin type 9 serine protease gene, low LDL cholesterol, and cancer incidence. Cancer Epidemiol Prev Biomarkers 2007; 16: 2455-8.

26. Nowak C, Ärnlöv J. A Mendelian randomization study of the effects of blood lipids on breast cancer risk. Nature communications 2018; 9: 3957.

27. Galabova G, Brunner S, Winsauer G, et al. Peptide-based anti-PCSK9 vaccines-an approach for long-term LDLC management. PloS One 2014; 9: e114469.

28. Fattori E, Cappelletti M, Surdo PL, et al. Immunization against proprotein convertase subtilisin-like/kexin type 9 lowers plasma LDL-cholesterol levels in mice. J Lipid Res 2012; 53: 1654-61.

29. Crossey E, Amar MJ, Sampson M, et al. A cholesterol-lowering VLP vaccine that targets PCSK9. Vaccine 2015; 33: 5747-55.

30. Landlinger C, Pouwer MG, Juno C, et al. The ATO4A vaccine against proprotein convertase subtilisin/kexin type 9 reduces total cholesterol, vascular inflammation, and atherosclerosis in APOE* 3 Leiden. CETP mice. Eur Heart 2017; 38: 2499-507.

31. Momtazi-Borojeni AA, Jaafari MR, Badiee A, Sahebkar A. Long-term generation of antiPCSK9 antibody using a nanoliposome-based vaccine delivery system. Atherosclerosis 2019; 283: 69-78.

32. Schneeberger A, Mandler M, Otava O, et al. Development of AFFITOPE vaccines for Alzheimer's disease (AD) - from concept to clinical testing. J Nutr Health Aging 2009; 13: 264-7.

33. Slingluff CL, Yamshchikov G, Neese P, et al. Phase I trial of a melanoma vaccine with gp100280-288 peptide and tetanus helper peptide in adjuvant: immunologic and clinical outcomes. Clin Cancer Res 2001; 7: 3012 24.

34. Bartlett GR. Phosphorus assay in column chromatography. J Biol Chem 1959; 234: 466-8.

35. Huang Z, Szoka Jr FC. Sterol-modified phospholipids: cholesterol and phospholipid chimeras with improved biomembrane properties. J Am Chem Soc 2008; 130: 15702-12.

36. Huang Z, Jaafari MR, Szoka Jr FC. Disterolphospholipids: nonexchangeable lipids and their application to liposomal drug delivery. Angewandte Chemie 2009; 121 : 4210-3.

37. Schluep T, Hwang J, Cheng J, et al. Preclinical efficacy of the camptothecin-polymer conjugate IT-101 in multiple cancer models. Clin Cancer Res 2006; 12: 1606-14.

38. Huang SK, Mayhew E, Gilani S, et al. Pharmacokinetics and therapeutics of sterically stabilized liposomes in mice bearing C-26 colon carcinoma. Cancer Res 1992; 52: 6774-81.
39. Schmidt AF, Holmes MV, Preiss D, et al. Phenome-wide association analysis of LDL-cholesterol lowering genetic variants in PCSK9. bioRxiv 2018; 329052. 